\title{
The formation of $\mathrm{cD}$ Halos: the case of NGC 3311 in the Hydra I Cluster
}

\author{
Ventimiglia G. ${ }^{1}$, Gerhard O. ${ }^{1}$ and Arnaboldi M. ${ }^{2}$
}

${ }^{1}$ Max-Planck-Institut fuer Ex. Physik, Giessenbachstrasse D-85748, Garching b. Muenchen

${ }^{2}$ European Organisation for Astronomical Research in the Southern Hemisphere, Karl-Schwarzschild-Strasse 2, D-85748 Garching b. Muenchen

We have studied the core of the Hydra I cluster, around its central cD galaxy, NGC 3311. We have analyzed the kinematics a sample of 60 intracluster planetary nebulae (PNs), detected using the multi-slit imaging spectroscopy technique (MSIS, Gerhard et al., 2005, Ventimiglia et al., 2008). PNs are good tracers of light (Coccato et al., 2009) and the MSIS allows to measure their velocities and positions at the same time. The histogram of the PN radial velocities presents several discrete components. We are comparing this result with $\Lambda \mathrm{CDM}$ hydro-dynamical simulations and other data in order to interpret it in the framework of the formation of extended halos around $\mathrm{cD}$ galaxies. $\mathrm{V}$ band photometric data around NGC 3311 have revealed the presence of an excess of light in the North-East part of the galaxy, which is spatially coincident with most of the PNs contributing to the reddest peak in the PNs LOSVD. We have measured, using Long-Slit data, the velocity of HCC26, a dwarf (DW) galaxy in the middle of the excess of light. The reddest peak in the PNs LOSVD is consistent both with the velocity of HCC 26 and of several other DWs in the same region. We are investigating the possibility that the light in excess has been stripped from these galaxies and now incorporated into the halo of NGC 3311.

\section{References}

Coccato, L., et al. 2009, MNRAS 394, 1249C

Gerhard, O., et al. 2005, ApJ 621, L93

Ventimiglia, G., Arnaboldi, M., \& Gerhard, O. 2008, AN 329, 1057 